\title{
APOC3 rs2070667 Associates with Serum Triglyceride Profile and Hepatic Inflammation in Nonalcoholic Fatty Liver Disease
}

\author{
Qing-Yang Xu $\mathbb{D},{ }^{1}$ Han Li $\mathbb{D}^{1},{ }^{1}$ Hai-Xia Cao $\mathbb{D},{ }^{1}$ Qin Pan $\mathbb{D}^{1},{ }^{1}$ and Jian-Gao Fan $\mathbb{D}^{1,2,3}$ \\ ${ }^{1}$ Department of Gastroenterology, Xinhua Hospital, School of Medicine, Shanghai Jiaotong University, Shanghai 200092, China \\ ${ }^{2}$ Department of Pediatric Gastroenterology, Xinhua Hospital, Shanghai Jiaotong University School of Medicine, \\ Shanghai 200092, China \\ ${ }^{3}$ Shanghai Key Laboratory of Children's Digestion and Nutrition, Shanghai 200092, China
}

Correspondence should be addressed to Qin Pan; panqin@xinhuamed.com.cn and Jian-Gao Fan; fanjiangao@xinhuamed.com.cn Received 1 September 2020; Revised 2 November 2020; Accepted 10 November 2020; Published 27 November 2020

Academic Editor: Zhanjun Jia

Copyright (C) 2020 Qing-Yang Xu et al. This is an open access article distributed under the Creative Commons Attribution License, which permits unrestricted use, distribution, and reproduction in any medium, provided the original work is properly cited.

\begin{abstract}
Single-nucleotide polymorphisms (SNPs) of apolipoprotein C3 (APOC3) play important role in lipid metabolism, and dyslipidemia underlies nonalcoholic fatty liver disease (NAFLD). But the correlation of serum lipidomics, APOC3 SNPs, and NAFLD remains limited understood. Enrolling thirty-four biopsy-proven NAFLD patients from Tianjin, Shanghai, Fujian, we investigated their APOC3 genotype and serum lipid profile by DNA sequencing and ultraperformance liquid chromatography-tandem mass spectrometry (UPLC-MS/MS), respectively. Scoring of hepatocyte steatosis, ballooning, lobular inflammation, and liver fibrosis was then performed to reveal the role of lipidomics-affecting APOC3 SNPs in NAFLD-specific pathological alterations. Here, we reported that APOC3 SNPs (rs4225, rs4520, rs5128, rs2070666, and rs2070667) intimately correlated to serum lipidomics in NAFLD patients. A allele instead of $G$ allele at rs2070667, which dominated the SNPs underlying lipidomic alteration, exhibited downregulatory effect on triacylglycerols (TGs: TG 54:7, TG 54:8, and TG 56:9) containing polyunsaturated fatty acid (PUFA). Moreover, subjects with low-level PUFA-containing TGs were predisposed to high-grade lobular inflammation (TG 54:7, rho $=-0.454$ and $P=0.007$; TG 54:8, rho $=-0.411$ and $P=0.016$; TG 56:9, rho $=-0.481$ and $P=0.004$ ). The significant correlation of $A P O C 3$ rs2070667 and inflammation grading [G/G vs. G/A+A/A: $0.00(0.00$ and 1.00$)$ vs. 1.50 (0.75 and 2.00), $P=0.022$ ] further confirmed its pathological action on the basis of lipidomics-impacting activity. These findings suggest an inhibitory effect of A allele at APOC3 rs2070667 on serum levels of PUFA-containing TGs, which are associated with high-grade lobular inflammation in NAFLD patients.
\end{abstract}

\section{Introduction}

Nonalcoholic fatty liver disease (NAFLD) reflects an important pathological syndrome demonstrating hepatocyte steatosis, ballooning, and lobular inflammation, with clinical outcomes of liver fibrosis/cirrhosis, hepatocellular carcinoma, and related mortality [1-8]. By its rapid growing incidence in the recent decades, NAFLD has already been a leading cause of chronic liver diseases worldwide [9-13]. Aberrant lipid metabolism, which facilitates the hepatocyte steatosis, oxidative stress, hepatic injury, and inflammation $[12,14-16]$, is now widely accepted to serve as a fundamental etiology that results in the NAFLD-inducing "multiple hits" $[10,17,18]$.
Nowadays, multiple studies reveal an intimate association of single-nucleotide polymorphisms (SNPs) in the lipometabolism-related genes [19-24] and the genetic susceptibility of NAFLD [25-32]. Among these genes, APOC3 encodes apolipoprotein C3 (apoC3), which is the critical inhibitor of lipoprotein lipase (LPL) in chylomicron (CM) remnants and very-low-density lipoprotein (VLDL) [3335]. The modulatory effect of apoC3 on LDL receptor (LDLR) activation causes the reduction of uptake and integration of triglyceride- (TG-) rich particles in hepatocytes [33-35]. Loss-of-function mutations of APOC3 (R19X, IVS2 $+1 \mathrm{G} \rightarrow \mathrm{A}, \mathrm{IVS} 3+1 \mathrm{G} \rightarrow \mathrm{T}, \mathrm{A} 43 \mathrm{~T}$, and V50M) $[33,36]$ have been well described to correlate to plasma triglycerides [37] and related cardiovascular diseases (coronary heart disease, 
ischemic cardiovascular disease, etc.) in heterozygous carriers $[33,36]$. The critical role of APOC 3 in lipid metabolism, together with the dyslipidemia caused by APOC3 mutations $[38,39]$ and apolipoprotein C3 deficiency $[33,36]$, suggests an association of $A P O C 3$ SNPs and dyslipidemia-based NAFLD. However, this APOC3 SNPs and NAFLD association remains limited, yet controversial, understood [32, 40-43]. In brief, APOC3 variants (C-482T or T-455C) in the promoter are reported to predispose subjects to NAFLD by Petersen et al. [32], but this observation was disproved by Kozlitina et al. [41]. Thus, the correlation of APOC3 SNPs, serum lipids, and NAFLD needs further exploration.

We, therefore, performed APOC3 sequencing, serum lipidomic detection, and pathological evaluation in Chinese Han patients with biopsy-proven NAFLD. The association among APOC3 SNPs, serum lipid profile, and pathological scoring was then investigated to uncover the lipidomics-based intervention of APOC3 polymorphisms on NAFLD.

\section{Materials and Methods}

2.1. Study Subjects. Thirty-four (male : female =19:15) Chinese Han patients with biopsy-proven NAFLD were recruited from Xinhua Hospital, Shanghai $(n=17)$; Tianjin Hospital of Infectious Diseases, Tianjin $(n=9)$; and Zhengxing Hospital, Zhangzhou, Fujian $(n=8)$ during January 2012 and June 2013 in this cross-sectional study (Table 1). The following criteria were employed for patient exclusion: (1) drinking history or excessive alcohol consumption, (2) virus hepatitis, (3) steatosis-related chronic liver diseases, and (4) liver transplantation [44-47]. Each participator provides $1 \mathrm{~mL}$ total blood for further detection. Institutional approval of this study was obtained from Xinhua Hospital Research Ethics Committee, and informed consent was issued by each participant. All methods in this study were conducted in accordance with the approved guidelines and the Declaration of Helsinki.

2.2. DNA Isolation and Genotyping of APOC3 SNPs. After centrifugation of $500 \mu \mathrm{l}$ total blood, DNA was extracted from peripheral mononuclear cells by QiAamp DNA Mini Kit (Qiagen, Venlo, Netherlands). The concentration and quality of DNA were verified using NanoDrop ${ }^{\circledR}$ ND-1000 (Thermo Fisher Scientific, Waltham, MA, USA) and $0.8 \%$ agarose gel electrophoresis. Thereafter, primers of five APOC3 SNPs (rs4225, rs4520, rs5128, rs2070666, and rs2070667) were designed on the basis of dbSNP database (https://www.ncbi .nlm.nih.gov/snp/) to construct a custom Ion AmpliSeq panel (Thermo Fisher Scientific, Waltham, MA, USA). The emulation polymerase chain reaction (PCR) of the template DNA was processed using the Ion OneTouch 2 System (Thermo Fisher Scientific, Waltham, MA, USA) according to the manufacturer's instructions. APOC3 SNPs were successively genotyped according to the following procedures: (1) DNA sequencing by Ion 318 Chip on the Ion PGM ${ }^{\mathrm{TM}}$ System (Thermo Fisher Scientific, Waltham, MA, USA) and (2) data analysis by the Auto-user software (Life Technology, Gaithersburg, MD, USA) [48].
TABLE 1: Basic information of population.

\begin{tabular}{lcc}
\hline & Mean & SD \\
\hline Age (years) & 41.03 & 14.81 \\
Weight (kg) & 75.34 & 9.49 \\
Waist (cm) & 90.59 & 6.61 \\
BMI (kg/m2) & 26.90 & 3.13 \\
ALB (mg/dl) & 41.36 & 7.55 \\
ALT (U/L) & 70.47 & 56.19 \\
AST (U/L) & 52.04 & 34.78 \\
TC (mg/dl) & 4.70 & 0.64 \\
TG (mg/dl) & 1.71 & 0.70 \\
HDL (mg/dl) & 1.16 & 0.28 \\
LDL (mg/dl) & 2.70 & 0.56 \\
\hline
\end{tabular}

SD: standard deviation; ALB: albumin; ALT: alanine aminotransferase; AST: aspartate aminotransferase; TC: total cholesterol; TG: total triglyceride; HDL: high-density lipoprotein; LDL: low-density lipoprotein.

2.3. Lipidomics Analysis. Serum sample ( $40 \mu \mathrm{l})$ from each NAFLD patient was subjected to serum lipidomic analysis using Triple TOF 5600 mass spectrometer (AB SCIEX, Framingham, MA, USA) by means of untargeted ultraperformance liquid chromatography-tandem mass spectrometry (UPLC-MS/MS). Firstly, a Waters BEH C8 column $(2.1 \mathrm{~mm} \times 100 \mathrm{~mm}$ and $1.7 \mu \mathrm{m})$ was used for lipid separation. The mobile phases were composed of $3: 2(\mathrm{v} / \mathrm{v}) \mathrm{ACN} / \mathrm{H}_{2} \mathrm{O}$ (10 mM AcAm, phase A) and 9:1 (v/v) IPA/ACN (10 mM AcAm, phase B). A 20-minute elution gradient program was run at the flow rate of $0.26 \mathrm{~mL} / \mathrm{min}$ and the column temperature of $55^{\circ} \mathrm{C}$. The elution gradient stared with $32 \%$ B for $1.5 \mathrm{~min}$ and rose up linearly to $85 \% \mathrm{~B}$ at $14 \mathrm{~min}$, then reached $97 \% \mathrm{~B}$ at $15.5 \mathrm{~min}$ for $2.5 \mathrm{~min}$. It returned to $32 \% \mathrm{~B}$ within $0.1 \mathrm{~min}$ and held for $1.9 \mathrm{~min}$ for column equilibration.

The UPLC-MS/MS parameters conducted for lipid detection were summarized as follows: temperature of interface heater, $600^{\circ} \mathrm{C}$ in electrospray ionization (ESI) (-) and $500^{\circ} \mathrm{C}$ in ESI (+); ion spray voltage of MS: $4500 \mathrm{~V}$ in ESI (-) and $5500 \mathrm{~V}$ ESI (+); declustering potential: $100 \mathrm{~V}$ ESI $(-)$ and $100 \mathrm{~V}$ ESI (+); collision energy: $10 \mathrm{~V}$ ESI (-) and $10 \mathrm{~V}$ ESI $(+)$. Thirteen quality control (QC) samples were randomly inserted into the testing sequence.

Raw data obtained was identified using LipidView/PeakView (AB SCIEX, Framingham, MA, USA) and quantified using MultiQuant 2.0 (AB SCIEX, Framingham, MA, USA). The relative standard deviation of 239 serum lipids in QC samples was measured against the internal standards [49].

2.4. Hepatic Histopathologic Assessment. Liver specimens from each patient were obtained by ultrasound-guided needle biopsy. Each sample was then treated by $10 \%$ formalin fixing, paraffin embedding, slicing, and hematoxylin \& eosin (HE) and Masson's trichrome staining in succession. Three pathologists who were not aware of the study assessed the NAFLD-related pathological characteristics according to the steatosis, activity, and fibrosis (SAF) scoring method as follows: (1) steatosis (S0, <5\%; S1, 5-33\%; S2, 34-66\%; S3, $>66 \%$ ); (2) activity: sum of lobular inflammation ( 0 , no foci 
per $200 \times$ field; $1,<2$ foci per $200 \times$ field; $2,2-4$ foci per $200 \times$ field; $3,>4$ foci per $200 \times$ field) and ballooning (0, none; 1 , few balloon cells; 2, many cells/prominent ballooning); (3) fibrosis (F0, none; F1, perisinusoidal or portal fibrosis; F2, perisinusoidal and periportal fibrosis without bridging; F3, bridging fibrosis; F4, cirrhosis) [1, 50-52].

2.5. Statistical Analysis. All data were expressed as means \pm standard deviation (SD). Unpaired Student's independent $t$-test was used to investigate the differences in serum lipidomics after examining the normality of data $(P \geq 0.1)$ by Kolmogorov-Smirnov test. Spearman's correlation was performed to evaluate the association of serum lipid profile and hepatic histological parameters. Differences in histological parameters were analyzed by Mann-Whitney $U$ test. Analyses were performed using SPSS21.0 (SPSS Inc., Chicago, IL, USA) with a two-side significant criterion at $P<0.05$.

\section{Results}

3.1. APOC3 SNPs Correlated with Serum Lipidomics in NAFLD Patients. Detecting APOC3 SNPs and 239 serum lipids, the effect of APOC3 polymorphisms on serum lipidomics was subjected to evaluation. Dramatically, there was a distinct correlation between APOC3 SNPs (rs4225, rs4520, rs5128, rs2070666, and rs2070667) and serum lipidomics in our NAFLD cohort (Table 2). APOC3 SNP-related lipids, including ceramide (Cer), diacylglycerol (DG), choline plasmalogen (PCO), phosphatidylethanolamine (PE), ethanolamine plasmalogen (PEO), phosphatidylinositol (PI), and triacylglycerol (TG), characterized the lipidomic alteration (Table 2).

3.2. G/A or A/A Genotype of APOC3 rs2070667 Exhibited Downregulatory Effect on Serum Lipid Profile. In contrast to most APOC3 SNPs exhibiting limited-scale lipidomic association, APOC3 rs2070667 exerted wide-range impacts on serum lipids of Cer, DG, PCO, and TG with statistical significance (Table 2). When compared to those with G/G genotype, NAFLD patients carrying G/A+A/A genotypes at APOC3 rs2070667 demonstrated statistically lowered levels of TGs (TG 54:7, TG 54:8, and TG 54:9), whereas obvious higher levels of Cer (Cer 42:1;2), DG (DG 36:4), and PCO (PCO 38:4 and PCO 40:4) (Table 2).

3.3. APOC3 rs2070667-Dependent Low-Level TGs Associated with High-Grade Lobular Inflammation. To shed light on the interaction between serum lipidomics and NAFLD, we further investigated the association of APOC3 rs2070667related differential serum lipids and NAFLD-specific pathological disorders (hepatocyte steatosis, lobular inflammation, ballooning, and liver fibrosis). Interestingly, polyunsaturated fatty acid- (PUFA-) containing TGs among these ones showed a negative correlation with lobular inflammation in similar Spearman coefficients (TG 54:7, rho $=-0.454$ and $P=0.007$; TG 54:8, rho $=-0.411$ and $P=0.016$; TG 56:9, rho $=-0.481$ and $P=0.004$ ) (Figure $1(\mathrm{~b})$ ). Being compared to those carrying $\mathrm{G} / \mathrm{G}$ at $A P O C 3$ rs2070667, the NAFLD patients with G/A or A/A genotype were characterized by low serum levels of PUFA-containing TGs and high-grade lobular inflammation (Figure 1(b)). Other pathological characteristics of NAFLD, especially hepatocyte steatosis, shared the mild correlation of serum PUFA-containing TGs in an inverse manner (Figures 1(a), 1(c), and 1(d)).

3.4. NAFLD Patients Carrying A Allele at APOC3 rs2070667 Showed Severe Lobular Inflammation. By scoring hepatocyte steatosis, lobular inflammation, ballooning, and liver fibrosis, we assessed the role of APOC3 rs2070667 in NAFLD-related pathological characteristics. An aggravation of lobular inflammation and, to less extent, steatosis was documented in the NAFLD patients carrying A allele (G/A and A/A) at APOC3 rs2070667 in comparison to those with G allele (lobular inflammation: $G / G$ vs. G/A+A/A: 0.00 (0.00 and 1.00) vs. 1.50 ( 0.75 and 2.00$), P=0.022$; hepatocyte steatosis: G/G vs. G/A+A/A: 2.00 (1.00 and 2.00) vs. 2.00 (1.75 and $3.00), P=0.076$ ) (Figures $2(\mathrm{a})$ and $2(\mathrm{~b})$ ). However, different alleles at APOC3 rs2070667 showed insignificant effect on ballooning (G/G vs. G/A+A/A: 2.00 (1.00 and 2.00) vs. 1.50 (1.00 and 2.00), $P=0.744)$ and liver fibrosis $(G / G$ vs. G/A+A/A: 2.00 (1.00 and 3.00) vs. 1.00 (0.00 and 3.00), $P=$ 0.201 ) (Figures $2(\mathrm{c})$ and $2(\mathrm{~d})$ ). Thus, A allele at APOC3 rs2070667 is suggested to underlie severe lobular inflammation in the NAFLD patients with close association of lowered levels of PUFA-containing TGs.

\section{Discussion}

Multiple researches have shed light on the versatile actions of APOC 3 in lipid metabolism [35, 38, 39, 53-56]. apoC3 within $\mathrm{CM}$ is capable of inhibiting the LPL-dependent CM-TG hydrolysis and the hepatic CM intake on a basis of apoE and LDL receptor-related protein (LRP) combination [35, 53]. Upregulated apoC3 in VLDL also retards its clearance $[38,39,54-56]$. In addition, plasma level of apoC3 exhibited a correlation to HDL lipids of cholesterol ester (CE), TG, free cholesterol (FC), phosphatidylcholine (PC), PCO, sphingomyelin (SM), DG, Cer, and LPC [37]. This lipometabolic activity of apoC3 suggests an association of APOC3 SNPs and lipid metabolic traits [20, 23, 35, 37-39, 53-56].

Indeed, subjects with APOC3 -2854 G/T demonstrate higher serum TG [57], whereas $-482 \mathrm{C}>\mathrm{T}$ in $A P O C 3$ is related to the serum levels of TG and other lipids in the Chinese population [57]. An important role of polymorphisms tagging APOC3 is further convinced in the occurrence of dyslipidemia by Genome-Wide Association Studies (GWAS) [58-60]. Integrating DNA sequencing and UPLC-MS/MS analysis, our study presented novel findings that a group of APOC3 SNPs (rs4225, rs4520, rs5128, rs2070666, and rs2070667) exerted global impact on serum lipidomics in NAFLD patients. Moreover, APOC3 rs2070667 among these ones was statistically associated with 7 differential serum lipids, including Cer, DG, PCO, and TGs.

Nowadays, there are growing evidences highlighting a crucial effect of serum lipids on NAFLD [61-64]. Relying on the lipidomics-based evolutionary algorithm, serum lipids of TG $(16: 0,18: 0$, and $18: 1)$, PC (18:1 and $22: 6)$, and PCO $(24: 1$ and $20: 4)$ are put forward to be predictive biomarkers 


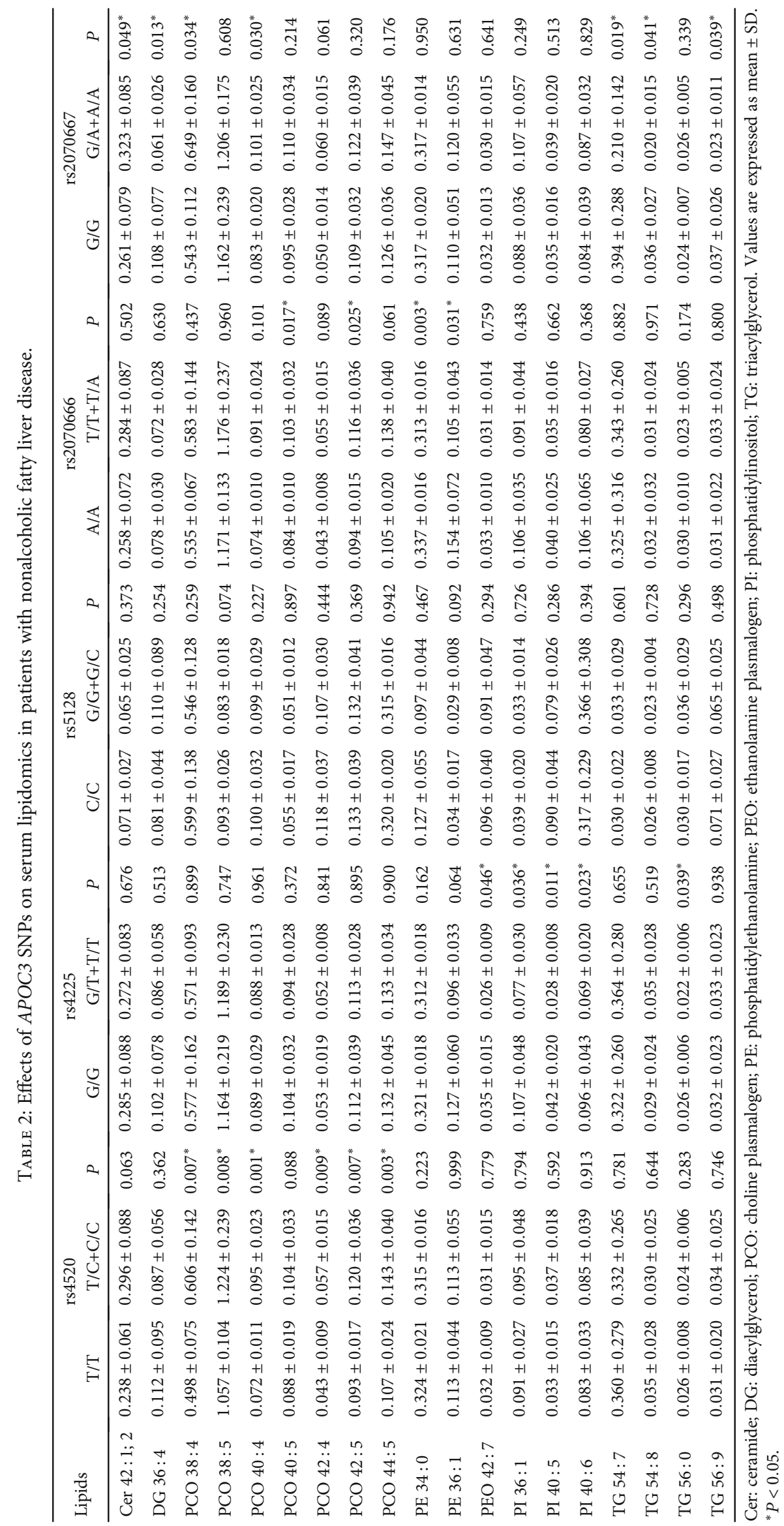



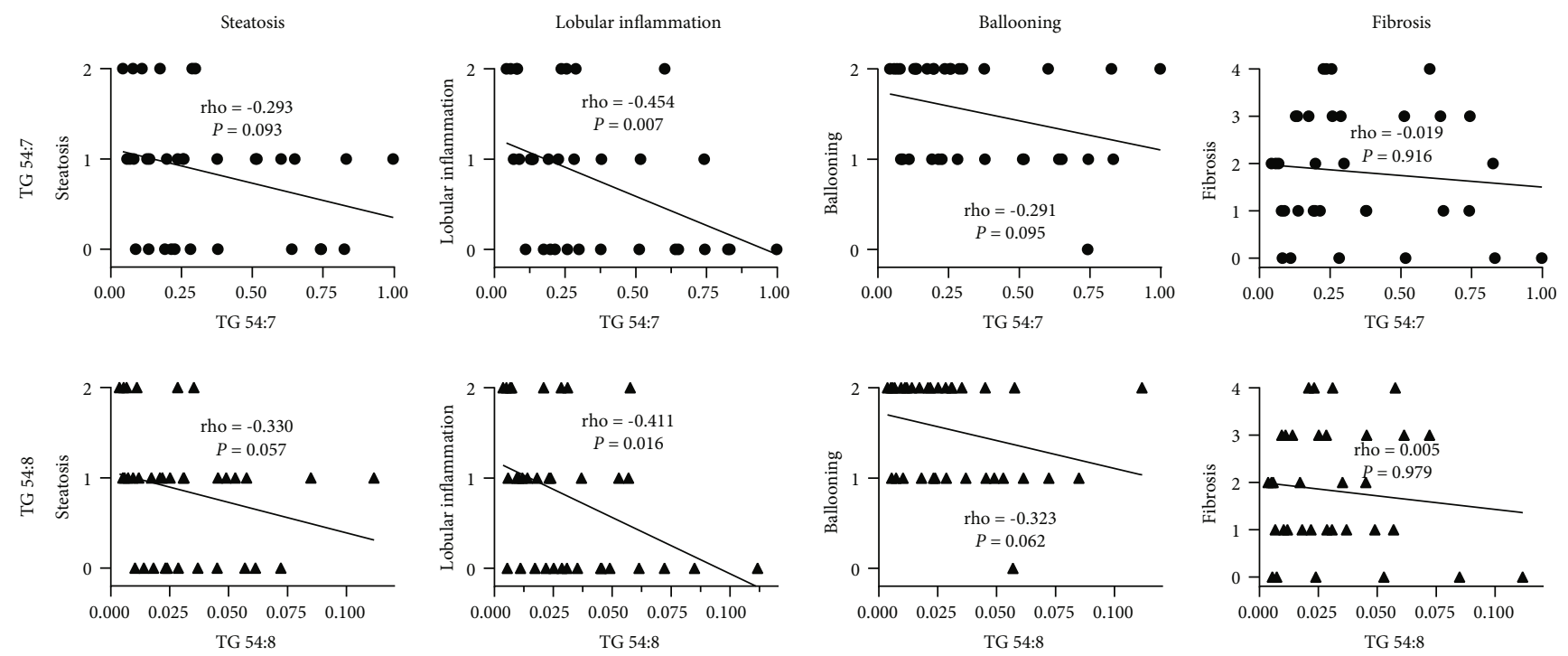

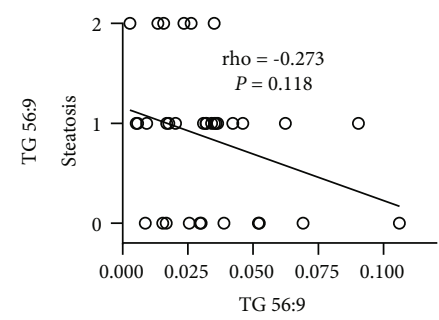

(a)

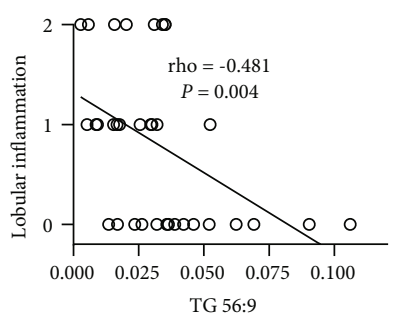

(b)

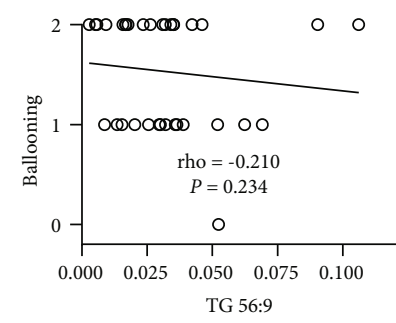

(c)

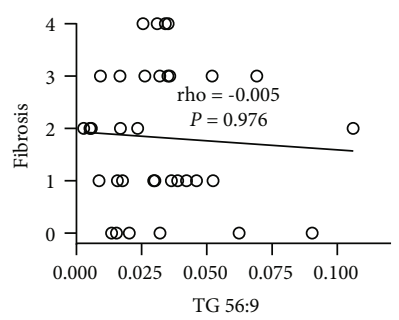

(d)

FIGURE 1: Lower-level TGs were correlated with higher-grade lobular inflammation in nonalcoholic fatty liver disease patients. Scatters reflected the serum levels of TGs (TG 54:7, TG 54:8, and TG 56:9) and pathologic scoring (steatosis, lobular inflammation, ballooning, and fibrosis) in patients with nonalcoholic fatty liver disease. Linear fit lines were presented with rho corresponding to the correlation of TGs and pathological characteristics. Inverse correlation between TG 54:7, TG 54:8, and TG 56:9 and lobular inflammation was observed with statistical significance. The rho values of fit lines in (b) were similar.

of NAFLD [61]. On the contrary, both serum LPCs and PUFA-containing phospholipids associate with the liver fat content in an inverse manner [61]. Decrease in serum palmitoyl-, stearoyl-, and oleoyl-LPC characterizes the mice with experimental nonalcoholic steatohepatitis (NASH) [62]. Nevertheless, FFA released from circulating TG and adipose tissue has been reported to contribute to hepatocellular FFA accumulation and steatosis [63, 64]. APOC3 SNPs, therefore, are proposed to interfere in the NAFLD by lipidomic modulation.

When compared to those with G/G genotype, we documented significantly lowered levels of TGs (TG 54:7, TG $54: 8$, and TG 54:9) in NAFLD patients with G/A or A/A genotype at $A P O C 3$ rs2070667. Moreover, there was a negative correlation between these PUFA-containing TGs and NAFLD-specific pathological characteristics, including lobular inflammation and steatosis, in comparable Spearman coefficients. In contrast to saturated fatty acids that upregulate the levels of proinflammatory cytokines, n-3 PUFAs have been described to attenuate the inflammation activity of liver by the reduction of proinflammatory cytokine (e.g., TNF- $\alpha$, IL- $\beta$, and IL-6) secretion, as well as the increase of antiinflammatory cytokine (e.g., adiponectin) [65-67]. Their incorporation into the phospholipids of inflammatory cells may underlie these pharmacological actions, resulting in the improved membrane fluidity and modified lipid derivatives [65]. On the other hand, PUFAs prevent the liver from steatosis on a basis of SREBP-1c and lipogenic gene (e.g., FAS, ACC, and SCD-1) downregulation [68] and then alleviate the hepatic inflammation by an amelioration of oxidative stress $[69,70]$.

In the present study, we verified much higher SAF grade of lobular inflammation in NAFLD patients carrying A allele at $A P O C 3$ rs 2070667 in comparison to those with $\mathrm{G}$ allele. By the mild increase in their steatosis scoring, an association was highlighted between APOC3 rs2070667 and hepatocyte steatosis. In result, APOC3 rs2070667 is indicated to be responsible for the deteriorated pathological characteristics in NAFLD patients by, to a large extent, its inhibitory impact on the serum levels of PUFA-containing TGs. Contrastively, there are some literatures deny the role of APOC3 SNPs in NAFLD $[40,71]$. This disagreement may be partially attributed to the test of blood lipids in routine method, which is insufficient to distinguish numerous components and their alterations in serum lipidomics.

Being contrast to most filtered SNPs locating in the exons of $A P O C 3$, rs 2070667 is found to be an intron-resided SNP with probably intact structure and catalytic activity of apoC3. Its effects on serum lipidomics and NAFLD could be attributed to the epigenetic regulations, such as DNA methylation 


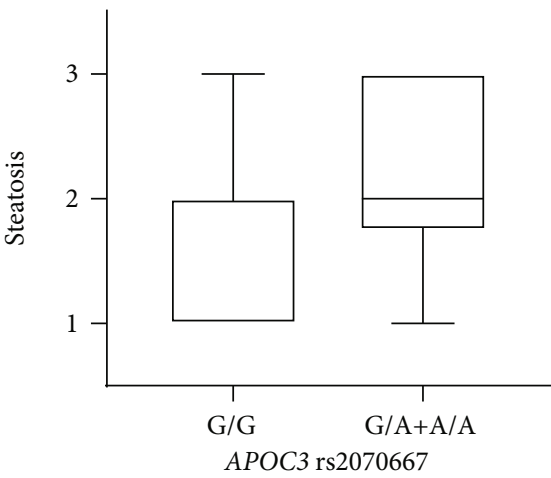

(a)

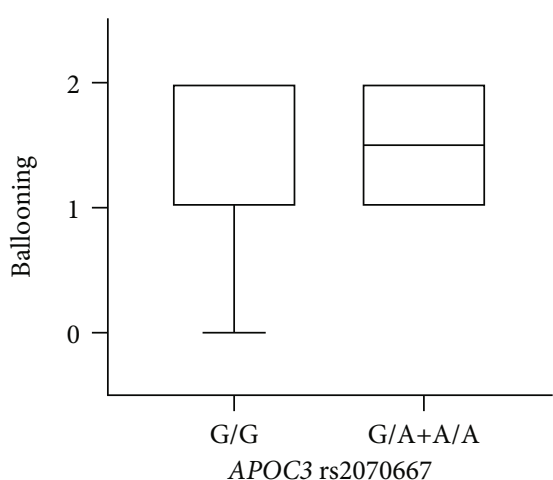

(c)

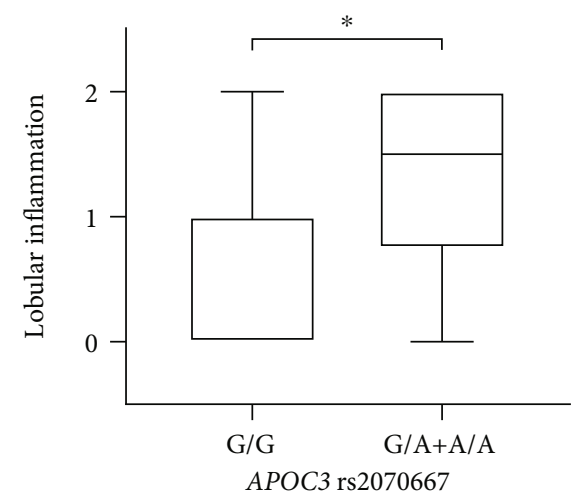

(b)

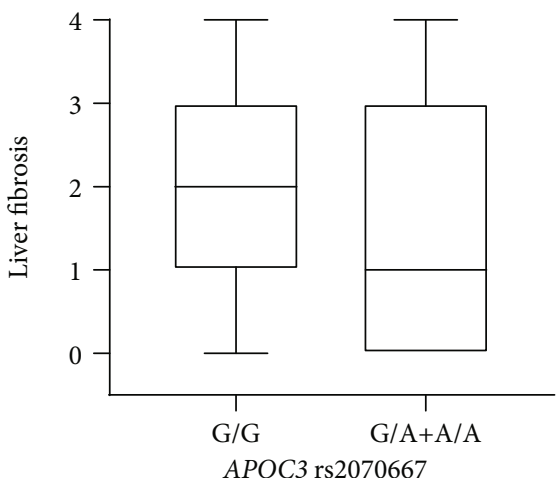

(d)

Figure 2: Nonalcoholic fatty liver disease patients carrying G/G vs. G/A+A/A genotype at APOC3 rs2070667 demonstrated higher-grade lobular inflammation. Box plots indicated the differences in pathological characteristics of steatosis, lobular inflammation, ballooning, and fibrosis between nonalcoholic fatty liver disease patients with $\mathrm{G} / \mathrm{G}$ vs. G/A+A/A genotype at APOC3 rs2070667. An aggravation of lobular inflammation was documented in the NAFLD patients carrying A allele (G/A and A/A) at APOC3 rs2070667 in comparison to those with G allele. Mild upregulated steatosis was also presented in the patients carrying A allele. Results were presented as medians and interquartile range. ${ }^{*} P<0.05$.

and miRNA-based expressive tuning [72-75]. Furthermore, transcriptional regulation reflects another potential mechanism of its lipidomic and pathological roles because approximately $40 \%$ of transcription factor (TF) binding sites have been identified in the introns [76].

However, there are some limitations in the present study. First, lacking of quantitative apoC3 test remains a shortcoming in highlighting the mechanisms underlying effects of APOC3 SNPs. In addition, untargeted UPLC-MS/MS instead of targeted UPLC-MS/MS was employed to investigate the effect of $A P O C 3$ on serum lipidomics. Thus, number of unsaturated bonds, but not their location, in serum lipids could be identified in our experiments.

\section{Conclusion}

APOC3 SNPs exhibit impact on the serum lipidomics of NAFLD patients. A allele at APOC3 rs2070667 demonstrates predominantly downregulatory effect on the serum lipid profile. Low-level PUFA-containing TGs $(54: 7,54: 8$, and $56: 9)$ among these differential lipids display significant association with high-grade lobular inflammation. Therefore, NAFLD patients carrying A instead of $\mathrm{G}$ allele at APOC3 rs2070667 may susceptible to hepatic inflammation upon the rs2070667-based alteration of serum TGs.

\section{Data Availability}

The data used to support the findings of this study are available from the corresponding author upon reasonable request.

\section{Conflicts of Interest}

The authors declare no conflict of interest.

\section{Authors' Contributions}

Qing-Yang $\mathrm{Xu}$ and Han Li contributed equally to this work.

\section{Acknowledgments}

This work is supported by the National Key R\&D Program of China (2017YFC0908903) and the National Natural Science Foundation of China (81270492, 81470859, and 81600464). We thank Guo-Wang Xu and Chun-Xiu Hu of Dalian Institute of Chemical Physics, Chinese Academy of Sciences.

\section{References}

[1] P. Bedossa, "Pathology of non-alcoholic fatty liver disease," Liver International, vol. 37, Supplement 1, pp. 85-89, 2017. 
[2] L. Castera, M. Friedrich-Rust, and R. Loomba, "Noninvasive assessment of liver disease in patients with nonalcoholic fatty liver disease," Gastroenterology, vol. 156, no. 5, pp. 12641281.e4, 2019.

[3] Y. Takahashi and T. Fukusato, "Histopathology of nonalcoholic fatty liver disease/nonalcoholic steatohepatitis," World Journal of Gastroenterology, vol. 20, no. 42, pp. 15539-15548, 2014.

[4] Q. M. Anstee, H. L. Reeves, E. Kotsiliti, O. Govaere, and M. Heikenwalder, "From NASH to HCC: current concepts and future challenges," Nature Reviews. Gastroenterology \& Hepatology, vol. 16, no. 7, pp. 411-428, 2019.

[5] C. D. Byrne and G. Targher, "NAFLD: a multisystem disease," Journal of Hepatology, vol. 62, no. 1, pp. S47-S64, 2015.

[6] A. Marengo, C. Rosso, and E. Bugianesi, "Liver cancer: connections with obesity, fatty liver, and cirrhosis," Annual Review of Medicine, vol. 67, no. 1, pp. 103-117, 2016.

[7] A. A. Kolodziejczyk, D. Zheng, O. Shibolet, and E. Elinav, “The role of the microbiome in NAFLD and NASH," EMBO Molecular Medicine, vol. 11, no. 2, 2019.

[8] A. Smith, K. Baumgartner, and C. Bositis, "Cirrhosis: diagnosis and management," American Family Physician, vol. 100, no. 12, pp. 759-770, 2019.

[9] Z. M. Younossi, A. B. Koenig, D. Abdelatif, Y. Fazel, L. Henry, and M. Wymer, "Global epidemiology of nonalcoholic fatty liver disease-meta-analytic assessment of prevalence, incidence, and outcomes," Hepatology, vol. 64, no. 1, pp. 73-84, 2016.

[10] E. Buzzetti, M. Pinzani, and E. A. Tsochatzis, "The multiple-hit pathogenesis of non-alcoholic fatty liver disease (NAFLD)," Metabolism-Clinical and Experimental, vol. 65, no. 8, pp. 1038-1048, 2016.

[11] G. Marchesini, C. P. Day, J. F. Dufour et al.et al., "EASLEASD-EASO Clinical Practice Guidelines for the management of non-alcoholic fatty liver disease," Journal of Hepatology, vol. 64, pp. 1388-1402, 2016.

[12] G. Musso, R. Gambino, and M. Cassader, "Recent insights into hepatic lipid metabolism in non-alcoholic fatty liver disease (NAFLD)," Progress in Lipid Research, vol. 48, no. 1, pp. 126, 2009.

[13] Z. M. Younossi, A. Baranova, K. Ziegler et al., "A genomic and proteomic study of the spectrum of nonalcoholic fatty liver disease," Hepatology, vol. 42, no. 3, pp. 665-674, 2005.

[14] H. Malhi and G. Gores, "Molecular mechanisms of lipotoxicity in nonalcoholic fatty liver disease," Seminars in Liver Disease, vol. 28, no. 4, pp. 360-369, 2008.

[15] Y. Kawano and D. E. Cohen, "Mechanisms of hepatic triglyceride accumulation in non-alcoholic fatty liver disease," Journal of Gastroenterology, vol. 48, no. 4, pp. 434-441, 2013.

[16] P. Puri, R. A. Baillie, M. M. Wiest et al., "A lipidomic analysis of nonalcoholic fatty liver disease," Hepatology, vol. 46, no. 4, pp. 1081-1090, 2007.

[17] Z. Hall, N. J. Bond, T. Ashmore et al., "Lipid zonation and phospholipid remodeling in nonalcoholic fatty liver disease," Hepatology, vol. 65, no. 4, pp. 1165-1180, 2017.

[18] H. Tilg and A. R. Moschen, "Evolution of inflammation in nonalcoholic fatty liver disease: the multiple parallel hits hypothesis," Hepatology, vol. 52, no. 5, pp. 1836-1846, 2010.

[19] C. Gieger, L. Geistlinger, E. Altmaier et al., "Genetics meets metabolomics: a genome-wide association study of metabolite profiles in human serum," PLoS Genetics, vol. 4, no. 11, article e1000282, 2008.
[20] D. M. Hallman, S. R. Srinivasan, W. Chen, E. Boerwinkle, and G. S. Berenson, "Longitudinal analysis of haplotypes and polymorphisms of the APOA5 and APOC3 genes associated with variation in serum triglyceride levels: the Bogalusa Heart Study," Metabolism, vol. 55, no. 12, pp. 1574-1581, 2006.

[21] M. Kanehisa, S. Goto, M. Hattori et al., "From genomics to chemical genomics: new developments in KEGG," Nucleic Acids Research, vol. 34, no. 90001, pp. D354-D357, 2006.

[22] S. Kathiresan, O. Melander, C. Guiducci et al., "Six new loci associated with blood low-density lipoprotein cholesterol, high-density lipoprotein cholesterol or triglycerides in humans," Nature Genetics, vol. 40, no. 2, pp. 189-197, 2008.

[23] G. P. Li, J. Y. Wang, S. K. Yan, B. S. Chen, H. Xue, and G. Wu, "Genetic effect of two polymorphisms in the apolipoprotein A5 gene and apolipoprotein C3 gene on serum lipids and lipoproteins levels in a Chinese population," Clinical Genetics, vol. 65, no. 6, pp. 470-476, 2004.

[24] K. C. Taylor, C. L. Carty, L. Dumitrescu et al., "Investigation of gene-by-sex interactions for lipid traits in diverse populations from the population architecture using genomics and epidemiology study," BMC Genetics, vol. 14, no. 1, p. 33, 2013.

[25] L. A. Adams, S. W. White, J. A. Marsh et al., "Association between liver-specific gene polymorphisms and their expression levels with nonalcoholic fatty liver disease," Hepatology, vol. 57, no. 2, pp. 590-600, 2013.

[26] N. Chalasani, X. Guo, R. Loomba et al., "Genome-wide association study identifies variants associated with histologic features of nonalcoholic fatty liver disease," Gastroenterology, vol. 139, no. 5, pp. 1567-1576.e6, 2010.

[27] T. Kitamoto, A. Kitamoto, M. Yoneda et al., "Genome-wide scan revealed that polymorphisms in the PNPLA3, SAMM50, and PARVB genes are associated with development and progression of nonalcoholic fatty liver disease in Japan," Human Genetics, vol. 132, no. 7, pp. 783-792, 2013.

[28] M. F. Feitosa, M. K. Wojczynski, K. E. North et al., "The ERLIN1CHUK-CWF19L1 gene cluster influences liver fat deposition and hepatic inflammation in the NHLBI Family Heart Study," Atherosclerosis, vol. 228, no. 1, pp. 175-180, 2013.

[29] X.-R. Shang, J.-Y. Song, F.-H. Liu, J. Ma, and H.-J. Wang, "GWAS-identified common variants with nonalcoholic fatty liver disease in Chinese children," Journal of Pediatric Gastroenterology and Nutrition, vol. 60, no. 5, pp. 669-674, 2015.

[30] E. K. Speliotes, L. M. Yerges-Armstrong, J. Wu et al., "Genome-wide association analysis identifies variants associated with nonalcoholic fatty liver disease that have distinct effects on metabolic traits," Plos Genetics, vol. 7, no. 3, article e1001324, 2011.

[31] M. R. Li, S. H. Zhang, K. Chao et al., "Apolipoprotein C3 $(-455 \mathrm{~T}>\mathrm{C})$ polymorphism confers susceptibility to nonalcoholic fatty liver disease in the Southern Han Chinese population," World Journal of Gastroenterology, vol. 20, no. 38, pp. 14010-14017, 2014.

[32] K. F. Petersen, S. Dufour, A. Hariri et al., "Apolipoprotein C3 gene variants in nonalcoholic fatty liver disease," The New England Journal of Medicine, vol. 362, no. 12, pp. 1082-1089, 2010.

[33] J. Crosby, G. Peloso, P. Auer et al., "Loss-of-function mutations in $A P O C 3$, triglycerides, and coronary disease," New England Journal of Medicine, vol. 371, no. 1, pp. 22-31, 2014.

[34] A. Onat, G. Hergenc, V. Sansoy et al., "Apolipoprotein C-III, a strong discriminant of coronary risk in men and a determinant 
of the metabolic syndrome in both genders," Atherosclerosis, vol. 168, no. 1, pp. 81-89, 2003.

[35] K. W. van Dijk, P. C. N. Rensen, P. J. Voshol, and L. M. Havekes, "The role and mode of action of apolipoproteins CIII and AV: synergistic actors in triglyceride metabolism?," Current Opinion in Lipidology, vol. 15, no. 3, pp. 239-246, 2004.

[36] A. B. Jorgensen, R. Frikke-Schmidt, B. G. Nordestgaard, and A. Tybjaerg-Hansen, "Loss-of-function mutations in APOC3 and risk of ischemic vascular disease," New England Journal of Medicine, vol. 371, no. 1, pp. 32-41, 2014.

[37] M. Ståhlman, B. Fagerberg, M. Adiels et al., "Dyslipidemia, but not hyperglycemia and insulin resistance, is associated with marked alterations in the HDL lipidome in type 2 diabetic subjects in the DIWA cohort: impact on small HDL particles," Biochimica et Biophysica Acta, vol. 1831, no. 11, pp. 16091617, 2013.

[38] D. M. Waterworth, J. Ribalta, V. Nicaud, J. Dallongeville, S. E. Humphries, and P. Talmud, "ApoCIII gene variants modulate postprandial response to both glucose and fat tolerance tests," Circulation, vol. 99, no. 14, pp. 1872-1877, 1999.

[39] N. Maeda, H. Li, D. Lee, P. Oliver, S. H. Quarfordt, and J. Osada, "Targeted disruption of the apolipoprotein C-III gene in mice results in hypotriglyceridemia and protection from postprandial hypertriglyceridemia," Journal of Biological Chemistry, vol. 269, no. 38, pp. 23610-23616, 1994.

[40] X. Cheng, J. Yamauchi, S. Lee et al., “APOC3 protein is not a predisposing factor for fat-induced nonalcoholic fatty liver disease in mice," Journal of Biological Chemistry, vol. 292, no. 9, pp. 3692-3705, 2017.

[41] J. Kozlitina, E. Boerwinkle, J. C. Cohen, and H. H. Hobbs, "Dissociation between APOC3 variants, hepatic triglyceride content and insulin resistance," Hepatology, vol. 53, no. 2, pp. 467-474, 2011.

[42] H. Y. Lee, A. L. Birkenfeld, F. R. Jornayvaz et al., “Apolipoprotein CIII overexpressing mice are predisposed to diet-induced hepatic steatosis and hepatic insulin resistance," Hepatology, vol. 54, no. 5, pp. 1650-1660, 2011.

[43] A. Verrijken, S. Beckers, S. Francque et al., "A gene variant of PNPLA3, but not of APOC3, is associated with histological parameters of NAFLD in an obese population," Obesity, vol. 21, no. 10, pp. 2138-2145, 2013.

[44] P. Vajro, S. Lenta, P. Socha et al., "Diagnosis of nonalcoholic fatty liver disease in children and adolescents: position paper of the ESPGHAN Hepatology Committee," Journal of Pediatric Gastroenterology and Nutrition, vol. 54, no. 5, pp. 700713, 2012.

[45] Z. M. Younossi, P. Golabi, L. de Avila et al., "The global epidemiology of NAFLD and NASH in patients with type 2 diabetes: a systematic review and meta-analysis," Journal of Hepatology, vol. 71, no. 4, pp. 793-801, 2019.

[46] K. Kazankov, S. M. D. Jorgensen, K. L. Thomsen et al., “The role of macrophages in nonalcoholic fatty liver disease and nonalcoholic steatohepatitis," Nature Reviews Gastroenterology \& Hepatology, vol. 16, no. 3, pp. 145-159, 2019.

[47] R. S. Khan, F. Bril, K. Cusi, and P. N. Newsome, "Modulation of insulin resistance in nonalcoholic fatty liver disease," Нераtology, vol. 70, no. 2, pp. 711-724, 2019.

[48] Q. Pan, R. N. Zhang, Y. Q. Wang et al., "Linked PNPLA3 polymorphisms confer susceptibility to nonalcoholic steatohepatitis and decreased viral load in chronic hepatitis B," World Journal of Gastroenterology, vol. 21, no. 28, pp. 8605-8614, 2015.
[49] R.-X. Yang, C.-X. Hu, W.-L. Sun et al., "Serum monounsaturated triacylglycerol predicts steatohepatitis in patients with non-alcoholic fatty liver disease and chronic hepatitis B," Scientific Reports, vol. 7, no. 1, pp. 10517-10517, 2017.

[50] P. Bedossa and the FLIP Pathology Consortium, "Utility and appropriateness of the fatty liver inhibition of progression (FLIP) algorithm and steatosis, activity, and fibrosis (SAF) score in the evaluation of biopsies of nonalcoholic fatty liver disease," Hepatology, vol. 60, no. 2, pp. 565-575, 2014.

[51] P. Bedossa, C. Poitou, N. Veyrie et al., "Histopathological algorithm and scoring system for evaluation of liver lesions in morbidly obese patients," Hepatology, vol. 56, no. 5, pp. 17511759, 2012.

[52] D. E. Kleiner, E. M. Brunt, M. van Natta et al., "Design and validation of a histological scoring system for nonalcoholic fatty liver disease," Hepatology, vol. 41, no. 6, pp. 1313-1321, 2005.

[53] N. S. Shachter, "Apolipoproteins C-I and C-III as important modulators of lipoprotein metabolism," Current Opinion in Lipidology, vol. 12, no. 3, pp. 297-304, 2001.

[54] G. D. Norata, S. Tsimikas, A. Pirillo, and A. L. Catapano, "Apolipoprotein C-III: from pathophysiology to pharmacology," Trends in Pharmacological Sciences, vol. 36, no. 10, pp. 675-687, 2015.

[55] E. M. M. Ooi, P. H. R. Barrett, D. C. Chan, and G. F. Watts, "Apolipoprotein C-III: understanding an emerging cardiovascular risk factor," Clinical Science, vol. 114, no. 10, pp. 611$624,2008$.

[56] M. C. Jong, M. H. Hofker, and L. M. Havekes, "Role of ApoCs in lipoprotein metabolism: functional differences between ApoC1, ApoC2, and ApoC3," Arteriosclerosis Thrombosis and Vascular Biology, vol. 19, no. 3, pp. 472-484, 1999.

[57] C. Q. Lai, L. D. Parnell, and J. M. Ordovas, "The APOA1/C3/A4/A5 gene cluster, lipid metabolism and cardiovascular disease risk," Current Opinion in Lipidology, vol. 16, no. 2, pp. 153-166, 2005.

[58] S. Kathiresan, C. J. Willer, G. M. Peloso et al., "Common variants at 30 loci contribute to polygenic dyslipidemia," Nature Genetics, vol. 41, no. 1, pp. 56-65, 2009.

[59] H. Schunkert, I. R. König, S. Kathiresan et al., "Large-scale association analysis identifies 13 new susceptibility loci for coronary artery disease," Nature Genetics, vol. 43, no. 4, pp. 333-338, 2011.

[60] T. R. Webb, J. Erdmann, K. E. Stirrups et al., "Systematic evaluation of pleiotropy identifies 6 further loci associated with coronary artery disease," Journal of the American College of Cardiology, vol. 69, no. 7, pp. 823-836, 2017.

[61] M. Orešič, T. Hyötyläinen, A. Kotronen et al., "Prediction of non-alcoholic fatty-liver disease and liver fat content by serum molecular lipids," Diabetologia, vol. 56, no. 10, pp. 2266-2274, 2013.

[62] N. Tanaka, T. Matsubara, K. W. Krausz, A. D. Patterson, and F. J. Gonzalez, "Disruption of phospholipid and bile acid homeostasis in mice with nonalcoholic steatohepatitis," Нераtology, vol. 56, no. 1, pp. 118-129, 2012.

[63] E. Pardina, J. A. Baena-Fustegueras, R. Catalán et al., "Increased expression and activity of hepatic lipase in the liver of morbidly obese adult patients in relation to lipid content," Obesity Surgery, vol. 19, no. 7, pp. 894-904, 2009.

[64] J. Westerbacka, M. Kolak, T. Kiviluoto et al., "Genes involved in fatty acid partitioning and binding, lipolysis, monocyte/macrophage recruitment, and inflammation are overexpressed in 
the human fatty liver of insulin-resistant subjects," Diabetes, vol. 56, no. 11, pp. 2759-2765, 2007.

[65] P. Silva Figueiredo, A. Carla Inada, G. Marcelino et al., "Fatty acids consumption: the role metabolic aspects involved in obesity and its associated disorders," Nutrients, vol. 9, no. 10, p. 1158, 2017.

[66] K. Yamada, E. Mizukoshi, H. Sunagozaka et al., "Response to importance of confounding factors in assessing fatty acid compositions in patients with non-alcoholic steatohepatitis," Liver International, vol. 35, no. 6, p. 1773, 2015.

[67] K. M. Ajuwon and M. E. Spurlock, "Palmitate activates the NFkappaB transcription factor and induces IL-6 and TNFalpha expression in 3T3-L1 adipocytes," The Journal of Nutrition, vol. 135, no. 8, pp. 1841-1846, 2005.

[68] N. Tanaka, X. Zhang, E. Sugiyama et al., "Eicosapentaenoic acid improves hepatic steatosis independent of PPAR $\alpha$ activation through inhibition of SREBP-1 maturation in mice," Biochemical Pharmacology, vol. 80, no. 10, pp. 1601-1612, 2010.

[69] L. Liu, Q. Hu, H. Wu et al., "Dietary DHA/EPA ratio changes fatty acid composition and attenuates diet-induced accumulation of lipid in the liver of ApoE(-/-) mice," Oxidative Medicine and Cellular Longevity, vol. 2018, Article ID 6256802, 12 pages, 2018.

[70] C. G. Dossi, G. S. Tapia, A. Espinosa, L. A. Videla, and A. D'Espessailles, "Reversal of high-fat diet-induced hepatic steatosis by n-3 LCPUFA: role of PPAR- $\alpha$ and SREBP-1c," The Journal of Nutritional Biochemistry, vol. 25, no. 9, pp. 977-984, 2014.

[71] J. Hyysalo, I. Stojkovic, A. Kotronen et al., "Genetic variation in PNPLA3 but not APOC3 influences liver fat in nonalcoholic fatty liver disease," Journal of Gastroenterology and Hepatology, vol. 27, no. 5, pp. 951-956, 2012.

[72] J. Imgenberg-Kreuz, J. Carlsson Almlöf, D. Leonard et al., "DNA methylation mapping identifies gene regulatory effects in patients with systemic lupus erythematosus," Annals of the Rheumatic Diseases, vol. 77, no. 5, pp. 736-743, 2018.

[73] R. G. Bradley, E. B. Binder, M. P. Epstein et al., "Influence of child abuse on adult depression: moderation by the corticotropin-releasing hormone receptor gene," Archives of General Psychiatry, vol. 65, no. 2, pp. 190-200, 2008.

[74] M. Shieh, N. Chitnis, P. Clark, F. B. Johnson, M. Kamoun, and D. Monos, "Computational assessment of miRNA binding to low and high expression HLA-DPB1 allelic sequences," Human Immunology, vol. 80, no. 1, pp. 53-61, 2019.

[75] M. Hecker, N. Boxberger, N. Illner et al., "A genetic variant associated with multiple sclerosis inversely affects the expression of CD58 and microRNA-548ac from the same gene," PLoS Genetics, vol. 15, no. 2, article e1007961, 2019.

[76] G. Euskirchen, T. E. Royce, P. Bertone et al., "CREB binds to multiple loci on human chromosome 22," Molecular and Cellular Biology, vol. 24, no. 9, pp. 3804-3814, 2004. 